\title{
Periodicity Algorithms for Partial Words
}

\author{
Florin Manea $^{(A, C)} \quad$ Robert Mercaş $^{(B)}$ Cătălin Tiseanu ${ }^{(C)}$ \\ ${ }^{(A)}$ Otto-von-Guericke-Universität Magdeburg, Fakultät für Informatik \\ PSF 4120, D-39016 Magdeburg, Germany \\ manea@iws.cs. uni-magdeburg.de \\ ${ }^{(B)}$ GRLMC, Departament de Filologies Romàniques, Universitat Rovira i Virgili, \\ Av. Catalunya 35, Tarragona, 43002, Spain, \\ robertmercas@gmail.com \\ ${ }^{(C)}$ Faculty of Mathematics and Computer Science, University of Bucharest, \\ Str. Academiei 14, RO-010014 Bucharest, Romania, \\ ctiseanu@gmail.com
}

\begin{abstract}
In this paper we investigate several periodicity-related algorithms for partial words. First, we show that all periods of a partial word of length $n$ are determined in $\mathcal{O}(n \log n)$, and provide algorithms and data structures that help us answer in constant time queries regarding the periodicity of their factors. For this we need a $\mathcal{O}\left(n^{2}\right)$ preprocessing time and a $\mathcal{O}(n)$ updating time, whenever the words are extended by adding a letter. In the second part we show that substituting letters of a word $w$ with holes, with the property that no two holes are too close to each other, to make it periodic can be done in optimal time $\mathcal{O}(|w|)$. Moreover, we show that inserting the minimum number of holes such that the word keeps the property can be done as fast.
\end{abstract}

Keywords: Combinatorics on Words, Periodicity, Partial Words.

\section{Introduction}

Periodicity is one of the most fundamental properties of words. Problems correlated to periodicity computation have applications in formal languages and automata theory, algorithmic combinatorics on words, data compression, string searching and pattern matching algorithms (see [6, 16, 2] and the references therein). The first idea of a fast algorithm identifying all periods of a word was given in [12], with a small flaw, together with the first time-space optimal string matching algorithm. In [9], Crochemore provides the first correct time-space optimal algorithm computing the periods of a word. This solution, and many other subsequent efficient solutions of the problem, rely heavily on the possibility of performing string matching in linear time and space.

For partial words, sequences that beside regular symbols contain some holes or "don't cares", the concept of periodicity was also deeply analyzed ([2] surveys most of the work in this area, and discusses the results obtained in comparison with the ones obtained for words). 
To start with, the problem of testing the primitivity of a partial word (i.e., partial words with no period that divides their length) was discussed in [3, 4], where partial solutions were proposed; similarly to the classical case, these solutions were based on matching algorithms. To this end, we recall that a fast pattern matching algorithm is provided in [8], starting from the ideas initiated in [11]. More precisely, deterministic pattern matching algorithms solving the problem in $\mathcal{O}(n \log n)$ are known (see also [7]).

The study of repetitions in partial words was initiated in [17]. A string is said to contain a repetition if it has consecutive factors compatible with the same full word. In [17], it is proved that over a binary alphabet there exist infinite partial words that are cube-free, which, in other words, means that for all factors the periods are greater than one third of the factor's length. In [5] the authors solve a conjecture regarding the minimum size alphabet needed to construct an infinite partial word that remains overlap-free even after an arbitrarily insertion of holes; that is, all factors of the infinite word have periods greater than half their length. As an important step of their proof, the authors use a $\mathcal{O}(n d)$ algorithm that determines if, after hole insertions such that between each two holes there are at least $d-1$ non-hole symbols, a word has a certain period.

Algorithms regarding freeness of factors of partial words were firstly discussed in [17, 10]. In [10] the authors construct data structures which enable them, after a preprocessing phase done in $\mathcal{O}\left(n^{2}\right)$, to answer queries regarding the freeness of their factors in constant time. Moreover, the authors provide a method to update the data structures, in $\mathcal{O}(n \log n)$ time, whenever a symbol is concatenated to the right end of the existing string, and still answer the queries in constant time.

This paper proposes a series of algorithms for some basic problems related to periodicity in partial words, more efficient than the already existing ones, and discuss possible generalizations of these problems. After presenting some basics regarding partial words and periodicity, in the end of this section, our paper continues with two main parts. First, in Section 2. results from [3, 4, 10] are extended and improved. We investigate how all periods of a partial word of length $n$ are determined in $\mathcal{O}(n \log n)$, and then we provide algorithms and data structures that help us answer in constant time queries regarding the periodicity of factors. Whenever the words are extended, we have a $\mathcal{O}\left(n^{2}\right)$ preprocessing time and a $\mathcal{O}(n)$ updating time. In Section 3. we give algorithms that identify ways of making a word periodic by substituting in optimal time some letters by holes, in a restricted ways, and improve the already mentioned results from [5].

While the results of Section 2. regard some natural algorithmical questions regarding the periodicity of words, it is worth noting that the results presented in Section 3. may become useful in the area combinatorics on words. For instance, one may be interested in constructing words that do not contain any periodic partial words, that have at least several symbols between their holes ([17, 5]); in other words, one looks for words in which we can randomly substitute symbols with holes, such that no two holes are too close, and they remain periodic. Theorem 3.1. from the aforementioned Section, enables us to test efficiently whether a given word verifies this property or not. Also, in [14, 15] the idea of producing partial words that verify some combinatorial properties from a given full words, by substituting symbols with holes, is discussed and possible connections with bio-informatics are established.

We continue by giving several basic definitions.

Let $A$ be a nonempty finite set of symbols called an alphabet. Each element $a \in A$ is called a letter. A full word over $A$ is a finite sequence of letters from $A$, while a partial word over $A$ 
is a finite sequence of letters from $A_{\diamond}=A \cup\{\diamond\}$, the alphabet $A$ extended with the hole symbol $\diamond$.

The length of a partial word $u$ is denoted by $|u|$ and represents the total number of symbols in $u$. The empty word is the sequence of length zero and is denoted by $\varepsilon$. A partial word $u$ is a factor of a partial word $v$ if $v=x u y$ for some $x, y$. We say that $u$ is a prefix of $v$ if $x=\varepsilon$ and a suffix of $v$ if $y=\varepsilon$. We denote by $u[i]$ the symbol at position $i$ in $u$, and by $u[i . . j]$ the factor of $u$ starting at position $i$ and ending at position $j$, consisting of the concatenation of the symbols $u[i], \ldots, u[j]$, where $1 \leq i \leq j \leq n$.

The powers of a partial word $u$ are defined recursively by $u^{0}=\varepsilon$ and for $n \geq 1, u^{n}=u u^{n-1}$. The period of a partial word $u$ over $A$ is a positive integer $p$ such that $u[i]=u[j]$ whenever $u[i], u[j] \in A$ and $i \equiv j \bmod p$. In such a case, we say $u$ is $p$-periodic. If $u$ is $p$-periodic and $|u|>p$ we say that $u$ is non-trivially periodic.

If $u$ and $v$ are two partial words of equal length, then $u$ is said to be contained in $v$, denoted by $u \subset v$, if $u[i]=v[i]$, for all $u[i] \in A$. Partial words $u$ and $v$ are compatible, denoted by $u \uparrow v$, if there exists $w$ such that $u \subset w$ and $v \subset w$.

A partial word $u$ is said to be $d$-valid, for some positive integer $d$, if $u[i . . i+d-1]$ contains at most one $\diamond$-symbol, for all $1 \leq i \leq|u|-d+1$.

For a complete view on the basic definitions regarding combinatorics on words and partial words we refer the reader to [6, 16, 2]. The basic definitions needed to follow the algorithms presented here are found in [13]; we just stress out that all time bounds provided in this paper hold on the unit-cost RAM model.

\section{Testing the periodicity of partial words}

In this section we approach the canonical problem related to the periodicity of partial words. In particular, we first present a series of algorithms that identify, efficiently, all the periods of a given partial word. Further, we follow an approach from [10] and discuss how one can efficiently build data structures that allow to answer in constant time queries asking if a factor of a given partial word is periodic, or asking which is the minimum period of a partial word. We also present a method to update these data structures when a new symbol is added to the initial word.

\subsection{Finding all the periods of a word}

First, let us remark that it is trivial to check in linear time if a given partial word is $p$-periodic, when $p$ is also given as input: for a partial word $w$, of length $n$, we simply have to check if there exists a symbol $a_{i}$ that contains all the symbols of the set $S_{i}=\{w[i+k p] \mid i+k p \leq n, k \in I N\}$, for all $i \leq n$.

On the other hand, testing whether an input partial word $w$, of length $n$, is non-trivially periodic can be done in $\mathcal{O}(n \log n)$ time. The idea of such an algorithm is quite simple. We observe that the partial word $w$, over $V$, is non-trivially periodic if and only if it is compatible with a word $u v u$, with $u, v \in V^{*}$ and $|u|>0$. But $w$ is compatible with a word $u v u$, as above, if and only if $w$ is compatible with a factor of the word $w\left[\left\lfloor\frac{n}{2}\right\rfloor+1 . . n\right] \diamond^{n-1}$. This is checked in $\mathcal{O}(n \log n)$ time using the pattern matching algorithm proposed in [8]. Of course, if the 
rightmost factor of $w\left[\left\lfloor\frac{n}{2}\right\rfloor+1 . . n\right] \diamond^{n-1}$ compatible with $w$ is $w[i . . n] \diamond^{i-1}$, then the maximum period of $w$ is $i-1$.

Further, we provide an algorithm that finds all periods of a given partial word $w$, of length $n$.

Let us first note that $w$ is $p$-periodic if and only if $w$ is compatible with a prefix of each of the words $w[i p+1 . . n] \diamond^{n}$, for all $i \in I N$ with $i p \leq n$. For the proof assume that $n=q p+r$, with $0 \leq r<p$. Indeed, if $w$ is $p$-periodic, then it is clear that for all $i$, such that $1 \leq i \leq q$, we have $w[1 . .(q-i) p]$ is compatible with $w[i p+1 . . q p], w[(q-i) p+1 . .(q-i) p+r]$ is compatible with $w[q p+1 . . n]$, and, finally, $w[k p+r+1 . . n]$ is compatible with $\diamond^{n-k p-r}$. This shows the direct implication. For the reverse implication note that, since $w$ is compatible with a prefix of each of the words $w[i p+1 . . n] \diamond^{n}$, where $i \in I N$ with $i p \leq n$, it follows that, $w[j p+1 . .(j+1) p]$ is compatible with $w\left[j^{\prime} p . .\left(j^{\prime}+1\right) p\right]$ and $w[j p+1 . . j p+r]$ is compatible with $w[q p+1 . . n]$, for all $j, j^{\prime}$ such that $1 \leq j, j^{\prime} \leq q$. Following this, one can easily obtain the existence of a full word $u$, of length $p$, that is compatible with $w[j p+1 . .(j+1) p]$, for all $j$ with $q \leq j \leq q$, and $u[1 . . r]$ compatible with $w[q p+1 . . n]$. Actually, this implies that $w$ is $p$-periodic.

The above remark suggests the following approach for finding the non-trivial periods of a word. As a first step, we find all the values $i$, with $1 \leq i \leq n$, such that $w$ is compatible with the factor $w[i . . n] \diamond^{i-1}$ of $w \diamond^{n}$. We store an array Occ with $n$ positions, such that $O c c[i]=1$, when $w$ is compatible with $w[i . . n] \diamond^{i-1}$, and $O c c[i]=0$, otherwise. The second step consists in identifying the positive numbers $p$ that fulfill $O c c[k p+1]=1$, for all $k$ such that $0 \leq k p \leq n$. As we have noted above, such a number $p$ is a period of $w$.

The first step of the above algorithm can be completed in $\mathcal{O}(n \log n)$ time by running the pattern matching algorithm from [8] to find all the occurrences of $w$ in $w \diamond^{n}$.

The second step can also be implemented in $\mathcal{O}(n \log n)$ time, but in a more involved manner. The main idea is the following: for each $i$ such that $O c c[i+1]=0$, we conclude that all its divisors cannot be periods of $w$.

Therefore, we compute and store, for all $i$ with $1 \leq i \leq n$, its divisors. The total number of the divisors we need to analyze is $\left(\sum_{i=1}^{n} \sigma(i)\right)$, where $\sigma(i)$ is the number of positive divisors of $i$. But $\sum_{i=1}^{n} \sigma(i)=\sum_{i=1}^{n}\lfloor n / i\rfloor \leq \sum_{i=1}^{n} n / i$, since we can count for every $i$ how many numbers it divides (that is $\lfloor n / i\rfloor)$. This means that $\sum_{i=1}^{n} \sigma(i) \in \mathcal{O}(n \log n)$, because $\left(\left(\sum_{i=1}^{n} \frac{1}{i}\right) /(\log n)\right)$ converges to a positive constant. So, we keep in an array $L$ the lists $L[i]$ of divisors of every $i \in\{1, \ldots, n\}$. This data structure will take $\mathcal{O}(n \log n)$ space and can be computed in $\mathcal{O}(n \log n)$ using a sieve method: we go through all the numbers $k$ from 1 to $n$ and add $i$ to the lists of divisors of the numbers $k, 2 k, \ldots,\lfloor n / k\rfloor k$.

Once we finish computing the array $L$ we do the following. We define an array Per with $n-$ 1 elements, and set initially $\operatorname{Per}[i]=1$ for all $i \in\{1, \ldots, n-1\}$. Then, for each $i \in\{0, \ldots, n-1\}$ such that $O c c[i+1]=0$ we set $\operatorname{Per}[j]=0$ for all the elements $j$ of the list $L[i]$ (that is, for every $j$ that divides $i$ ). Clearly, computing the array Per takes $\mathcal{O}(n \log n)$ time, and from $\operatorname{Per}[i]=1$ it follows that $\operatorname{Per}[k i]=1$ for all $k$ such that $1 \leq k i+1 \leq n$.

According to the above remarks, a number $p$ is a period of $w$ if and only if $\operatorname{Per}[p]=1$. Consequently, all periods of $w$ can be computed in $\mathcal{O}(n \log n)$ time.

This result is particularly useful in two applications: finding the minimal period of a partial word and deciding whether a word is primitive. We are aware of the claims and proofs that these problems can be solvable in linear time (see [4] and, respectively, [3]). However, the algorithms proposed in these papers are relying on the fact that one can find all factors of a partial word $w w$ 
that are compatible with $w$ in linear time, by extending to the case of partial words some string matching algorithms for full words, that work in linear time. The proof of this fact was not given formally, and we are not convinced that such results actually hold. We refer the reader, for instance, to the discussions in [11].

Our algorithm enables us to find the smallest period of a partial word $w$ in time $\mathcal{O}(n \log n)$. We compute the array $\operatorname{Per}$ for $w$, find the minimum $p$ with $\operatorname{Per}[p]=1$ and return it as the answer of the problem. In order to decide whether a partial word $w$ is primitive in $\mathcal{O}(n \log n)$ time, we compute the array Per for $w$ and check whether there exists a number $p$ such that $\operatorname{Per}[p]=1$ and $p$ divides $|w|$.

\subsection{Queries and Updates}

In the following, we are interested in how to construct data structures that allow us to answer in constant time to queries regarding the periodicity of the factors of a given partial word, and that are easily updated once the word is extended.

Consider the following problem

\section{Problem 1}

1. Given a partial word $w$, of length $n$, over an alphabet $V$, preprocess it in order to answer the following types of queries:

"Is $w[i . . j] p$-periodic?", denoted $\operatorname{per}(i, j, p)$, where $i \leq j, i, j, p \in\{1, \ldots, n\}$.

"Which is the minimum period of $w[i . . j]$ ?", denoted $\operatorname{minper}(i, j)$, where $i \leq j, i, j \in$ $\{1, \ldots, n\}$.

2. Consider the following update operation for a partial word $w$ : add a symbol $a \in V \cup\{\diamond\}$ to the right end of $w$, to obtain wa. Preprocess $w$ and define a method to update the data structures constructed during the preprocessing process, in order to answer in constant time per queries, for a word obtained after several update operations were applied to $w$.

We describe a solution for the first part of the problem, that can easily be adapted to solve the second part as well.

First define the $n \times n$ matrix $A$, where $i, l \in\{1, \ldots, n\}$, as follows:

$$
A[i][l]=\left\{\begin{array}{c}
\max \{k \mid 0<k \leq i, i-k \text { is divisible by } l, \text { and } w[k] \neq \diamond\}, \\
\quad \text { if the set is non-empty; } \\
i-\lfloor(i-1) / l\rfloor l, \text { otherwise. }
\end{array}\right.
$$

Basically, $A[i][l]$ equals $k$, if all the symbols $w[k+l], w[k+2 l], \ldots, w[i]$ are equal to $\diamond$ and $w[k] \neq \diamond$, or, if such a value $k$ does not exist, $A[i][l]$ equals the leftmost position $t$ of the word with $i-t$ divisible by $l$. This matrix can be computed by dynamic programming, in time $\mathcal{O}\left(n^{2}\right)$ :

$$
A[i][l]=\left\{\begin{array}{l}
i, \text { if } w[i] \neq \diamond \text { or } i \leq l ; \\
A[i-l][l], \text { otherwise. }
\end{array}\right.
$$

Now we show that the formula holds. It is not hard to see that if $w[i] \neq \diamond$ or if $i \leq l$, then we have $A[i][l]=i$. Assume that none of these conditions hold. We show that $A[i][l]=A[i-l][l]=$ $k \neq i$. There are several cases to be considered. If $w[k]=\diamond$, then all the symbols $w[k+t l]$ are equal to $\diamond$, for $t \in\{0, \ldots,(i-k) / l\}$. Since $i>l$ it follows that $A[i-l][l]=k$ as well. If $w[k] \neq \diamond$, 


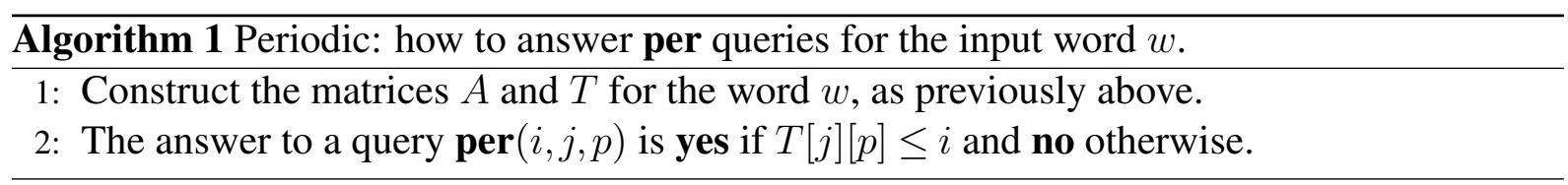

then all $w[k+t l]=\diamond$, for $t \in\{1, \ldots,(i-k) / l\}$. Furthermore, in this case $A[i-l][l]=k$, also, since the symbols $w[k+t l]$ are equal to $\diamond$, for $t \in\{1, \ldots,(i-l-k) / l\}$.

Now define the $n \times n$ matrix $T$, with $i, l \in\{1, \ldots, n\}$, as follows:

$$
T[i][l]=\min \{j \mid 0<j \leq i \text {, such that } w[j . . i] \text { is } l \text {-periodic }\} \text {. }
$$

We make a series of remarks that hold for all $i$ and $l$ :

- $T[i][l]=j>1$ if and only if $w[j . . i]$ is $l$-periodic and $w[j-1 . . i]$ is not;

- if $i \leq l$, then $T[i][l]=1$, since $w[1 . . i]$ is clearly $l$-periodic. Also, if $i>l$, then $w[i-l+1 . . i]$ is $l$-periodic. Thus, $i-T[i][l]+1 \geq l$. As a consequence we have that $T[1][l]=1$ and $i>T[i][l]$ if $i>1$;

- note that, for $i>1$, we have $T[i][l] \geq T[i-1][l]$. Indeed, if $w[p . . i]$ is $l$-periodic, then $w[p . . i-1]$ is also $l$-periodic. If $T[i][l]=T[i-1][l]$, then $i-T[i][l]+1>l$;

- the above remarks show that if $i>l$, then $i-T[i-1][l]+1>l$;

- let $i>l$ and $t=T[i-1][l]$. As shown above, we have $i-l \geq t$, and:

- if $A[i-l][l]<t$, then $w[t . . i]$ is periodic and $w[t-1 . . i]$ is not, since all symbols $w[j]$, where $i-j$ divisible by $l$ and $i>j \geq t$, are equal to $\diamond$ and $T[i][l] \geq t$. As a consequence $T[i][l]=T[i-1][l]$

- if $A[i-l][l] \geq t$ and $w[i] \uparrow w[A[i-l][l]]$, then all symbols $w[j]$, with $i-j$ divisible with $l$ and $i \geq j \geq t$, are contained in the same symbol. Thus, $w[t . . i]$ is $l$-periodic. As a consequence $T[i][l]=T[i-1][l]$

- if $A[i-l][l] \geq t$ and $w[i]$ is not compatible with $w[A[i-l][l]]$, then all symbols $w[j]$, where $i-j$ divisible by $l$ and $i \geq j>A[i-l][l]$, are contained in $w[i]$ and $w[A[i-l][l]+1 . . i]$ is $l$-periodic, while $w[A[i-l][l] . . i]$ is not $l$-periodic. Thus, $T[i][l]=A[i-l][l]+1$.

These remarks show that a matrix $T$ can be computed by dynamic programming using the following relation, that holds for all $i, l \in\{1, \ldots, n\}$ :

$$
T[i][l]=\left\{\begin{array}{l}
1, \text { if } i \leq l ; \\
T[i-1][l], \text { if } i>l \text { and } A[i-l][l]<T[i-1][l] \\
T[i-1][l], \text { if } i>l, A[i-l][l] \geq T[i-1][l] \text { and } w[i] \uparrow w[A[i-l][l]] \\
A[i-l][l]+1, \text { otherwise. }
\end{array}\right.
$$

Hence, the time needed to compute the elements of the matrix $T$ is $\mathcal{O}\left(n^{2}\right)$. Now, we use matrix $T$ to answer per queries, as described in Algorithm 1 .

Next we describe the way we deal with minper queries. We define the matrix $P_{m}$, with $n$ rows and $n$ columns, that stores the answer to our queries. Thus, for $i, j \in\{1, \ldots, n\}$ with $j \leq i$, we have:

$$
P_{m}[j][i]=\min \{p \mid w[j . . i] \text { is } p \text {-periodic }\} \text {. }
$$





Note that, whenever $j>i$, we have $P_{m}[j][i]=0$. Moreover, if $w[j . . i]$ is not $p$-periodic, for any $p<i-j+1$, then $P_{m}[j][i]=i-j+1$. Also, $P_{m}[j][i] \geq P_{m}[j+1][i]$. Consequently, the matrix $P_{m}$ can be computed using Algorithm2.

The time complexity for computing $P_{m}$, by Algorithm 2 is $\mathcal{O}\left(n^{2}\right)$. First, the construction of the matrices $A$ and $T$ can be done in quadratic time, as we have already shown. Next, the initialization of $P_{m}$ takes $\mathcal{O}\left(n^{2}\right)$ time, as well. Now, note that for a fixed $i$, the complete execution of the cycle in step 5 takes $\mathcal{O}(n)$ time. Indeed, in this cycle we set the values on one column of the matrix $P_{m}$. Since there are $n$ such values, we need exactly $n$ operations because we do not try to set a certain element of the matrix more than once. Consequently, the entire cycle 3 requires $\mathcal{O}\left(n^{2}\right)$ time, and this shows that the overall complexity of the algorithm is quadratic.

Algorithm 2 provides the setting to answer minper queries. This is depicted in Algorithm 3 .

Let us now describe how to solve the second part of Problem 1 when exactly one update is applied to a given partial word. If the word is updated more than once, we simply iterate this method. Suppose that we are given a partial word $w$, of length $n$, for which we compute the matrices $A$ and $T$, as previously described. Further, assume that we add to the partial word $w$ the symbol $a \in V \cup\{\diamond\}$ and obtain the word $w^{\prime}=w a$, where $w^{\prime}[n+1]=a$. Update the matrices $A$ and $T$, by adding to each of them a new column and a new row. One can easily observe that $A[i][n+1]=i$ and $T[i][n+1]=1$, for all $i \in\{1, \ldots, n+1\}$. On the other hand, the elements $A[n+1][l]$ and $T[n+1][l]$ can be computed using the already described recurrenceformulas, for all $l \in\{1, \ldots, n\}$. Also, one can add the new rows and columns to the matrices $T$ and $A$ in time $\mathcal{O}(n)$, see [10], and the computation of the newly inserted elements also requires $\mathcal{O}(n)$ time. Once all structures are updated, we answer per queries exactly as described in the previous section, at the second step of Algorithm 1 .

In order to update the matrix $P_{m}$, once $A$ and $T$ are updated, we proceed as follows. Firstly, set $P_{m}[n+1][n+1]=1$ and $P_{m}[n+1][i]=0$, for all $i \leq n$. Then, run the cycle from step 5 of Algorithm 2 for $i=n+1$, in order to compute the elements $P_{m}[j][n+1]$ with $j \leq n$. This takes, clearly, $\mathcal{O}(n)$ time. Of course, the answer to minper queries is still obtained as in step 2 of Algorithm 3 . 
To summarize, we are able to show the following:

A given partial word $w$, of length $n$, can be processed in time $\mathcal{O}\left(n^{2}\right)$ in order to be able to answer per and minper queries, in time $\mathcal{O}(1)$. If update operations, in which a symbol is added to the rightmost end of $w$, are applied, the previously constructed data structures are updated in at most $\mathcal{O}(n)$ time, per update, and both per and minper queries are answered in time $\mathcal{O}(1)$.

This represents an improvement of the results presented in [10]. In that paper the preprocessing time needed to answer in constant time queries asking whether a factor of a word is a $k$-repetition, $k$-free or overlap-free, where $k$ is a positive integer, is $\mathcal{O}\left(n^{2}\right)$, and the time needed for updating the data structures is $\mathcal{O}(n \log n)$ in a worst case analysis and $\mathcal{O}(n)$ in an amortized analysis. Moreover, several complicated data structures are used there. It is immediate how per queries can be used to answer, in $\mathcal{O}(1)$ time, queries asking whether a factor is a repetition, in fact a particular case of per queries, and, as shown in [10], all the other types of mentioned queries. Thus, following the reasoning in the afore mentioned paper, one can easily obtain that the preprocessing time needed to answer in constant time queries asking whether a factor of a word is a $k$-repetition, $k$-free or overlap-free, for some positive integer $k$, is $\mathcal{O}\left(n^{2}\right)$, and the time needed for updating the data structures is $\mathcal{O}(n)$ in the worst case. Furthermore, the data structures that we use here are quite simple.

Remark 1 Matrix $T$, computed for a partial word $w$, can also be used to determine the nontrivially periodic factors of the word. Basically, we compute for each $i$ the minimum $j$ such that $w[j . . i]$ is $p$-periodic, for some $p<i-j+1$, and store this value as $\min [i]$. The array min can be clearly computed in quadratic time from the matrix $T$. In particular, to compute min $[i]$ we compute the minimum of the set $P_{i}=\{T[i][l] \mid l \leq n\}$, which is done in linear time. Clearly, a factor $w\left[j^{\prime} . . i\right]$ is periodic if and only if $\min [i] \leq j^{\prime} \leq i$.

\subsection{Summary}

The results we obtained are the following:

Theorem 2.1 Let $w$ be a partial word of length $n$.

1. All the periods of $w$ can be computed in time $\mathcal{O}(n \log n)$.

2. The partial word $w$ can be processed in time $\mathcal{O}\left(n^{2}\right)$ in order to answer in constant time per and minper queries. After an update operation, the previously constructed data structures are updated in $\mathcal{O}(n)$ time, and both per and minper queries are answered in time $\mathcal{O}(1)$.

\section{From full words to periodic partial words}

In this section we change our focus to constructing, in linear time, a $p$-periodic partial word, starting from a full word, by replacing some of its symbols with holes such that no two consecutive holes are too close one to the other:

Problem 2 [5] Given a word $w \in V^{*}$, and positive integers $d$ and $p, d, p \leq|w|$, decide whether there exists a $p$-periodic $d$-valid partial word contained in $w$. 
The input of this problem consists of the word $w$ and the numbers $p$ and $d$. The alphabet $V$ can be arbitrarily large, but, clearly, we can assume that $|V| \leq n$ (that is, we do not care about symbols that do not appear in $w$ ).

We first approach a related problem.

Problem 3 Let $q$ and $m$ be positive integers, let $M$ be a sequence of $m$ arrays with at most $q$ elements from $V$, denoted $M_{1}, \ldots, M_{m}$, and let $d$ a positive integer, $d \leq m$. Furthermore, let $k \geq 1$ be a positive constant, and $p_{0}, \ldots, p_{k} \in\{1, \ldots, m\}$ such that $p_{0}=0, p_{k}=m$ and $p_{i}<p_{i+1}$, for $i \in\{0, \ldots, k-1\}$; assume that the arrays $M_{p_{i}+1}, \ldots, M_{p_{i+1}}$ have $q-i$ elements. Decide whether one can replace by holes several symbols of $M_{i}$ in order to obtain arrays $M_{i}^{\prime}$, where $i \in\{1, \ldots, m\}$, such that the following two conditions hold:

1. There exists a symbol $s_{i}$ that contains all the symbols of $M_{i}^{\prime}$ with $i \in\{1, \ldots, m\}$. This condition is called the periodicity condition.

2. For all $j \leq q$ and $i \in\{1, \ldots, m-d+1\}$, a multiset $\left\{M_{i}^{\prime}[j], \ldots, M_{i+d-1}^{\prime}[j]\right\}$ contains at most one hole. If one of the arrays $M_{k}^{\prime}$ in the set has less than $j$ elements, then the symbol $M_{k}^{\prime}[j]$ is missing from the set. This condition is called the $d$-validity condition.

The input consists of the sequence $M$ and the number $d$; again, the alphabet $V$ can be arbitrarily large, but we can assume that $|V| \leq m q$.

Intuitively, the solution of this problem is based on the following idea: a possible way to substitute some of the symbols of an array with holes, in order to fulfill the periodicity condition, induces some restrictions on the way the substitutions can be applied on the $d-1$ arrays that follow it (due to the $d$-validity condition). Thus, we obtain a set of restrictions for each $d$ consecutive arrays. We propose a formalization of the substitutions that can be made on each array as boolean variables and of the restrictions induced by these substitutions as formulas involving the variables. The fact that all the restrictions must be simultaneously fulfilled reflects in that the conjunction of all the formulas must be satisfiable. We show how the formulas can be constructed and how the satisfiability of their conjunction can be decided efficiently.

More formally, the solution of Problem 3 has two main steps. But before discussing them, note that there are two types of substitutions (also called replacements) that we can apply to the symbols of an array $M_{i}$ :

Type 1: We replace all the symbols $M_{i}[j] \neq M_{i}[1]$, with holes.

Type 2: There exists a symbol $M_{i}\left[j_{0}\right] \neq M_{i}[1]$ such that we replace all the symbols $M_{i}[j]$, different from $M_{i}\left[j_{0}\right]$, with holes.

Moreover, an array necessitates no substitution if all its symbols are equal.

The first step of our solution consists in defining the boolean variables $x_{i}$, for $1 \leq i \leq m$, where $x_{i}=1$ if and only if we need to apply a Type 2 replacement to $M_{i}$ to reach a valid solution. We derive, for each $d$ consecutive arrays of $M$, say $M_{i}, \ldots, M_{i+d-1}$, a boolean formula $\phi_{i}$, involving some of the variables defined above. Intuitively, the formula $\phi_{i}$ can be satisfied if and only if some of the symbols of the corresponding arrays can be replaced with holes and obtain a sequence that verifies both the periodicity condition and the $d$-validity condition.

As a final step, from all these formulas we construct a new formula $\phi_{M}$, which is true for an assignment of the variables $x_{i}$, with $1 \leq i \leq m$, if and only if Problem 3 has a solution (that can be obtained from this assignment). The formula $\phi_{M}$ has a quite simple form, and a solution for it can be easily obtained. 
We claim that all these steps can be implemented in $\mathcal{O}(m q)$ time. The time complexity does not depend on the cardinality of $V$ and the value of $d$.

Coming back to the solution of Problem 2, we show how it can be transformed in linear time $\mathcal{O}(|w|)$ into an instance of Problem 3, which can be solved also in time $\mathcal{O}(|w|)$; one can also give, effectively, the substitutions that must be performed in the word, in the same time complexity. Note, once more, that the complexity of our solutions depends only on $|w|$, and not on the cardinality of the input alphabet, nor on $d$, nor on $p$. Summarizing, we show:

Theorem 3.1 Problem 2 can be decided in linear time $\mathcal{O}(|w|)$. A solution for this Problem can be obtained in the same time complexity.

\subsection{A Linear Solution for Problem 3}

Here, we give some more technical details on the solution of Problem 3 .

For the sequence of arrays $M$, define the sequence of binary arrays $M^{b}$, with $M_{i}^{b}[j]=1$ if $M_{i}[j]=M_{i}[1]$, and $M_{i}^{b}[j]=0$ otherwise.

We observe there are two general types of replacements that can be applied to arrays $M_{i}$, in order to be sure that there exists a symbol $s_{i} \in V$ that contains all the elements of the modified array:

Type 1: We can replace all the symbols $M_{i}[j]$, different from $M_{i}[1]$, with holes. All symbols of the array are now contained in $M_{i}[1]$. Note that, in this case $M_{i}^{\prime}[j]=\diamond$, for all $M_{i}^{b}[j]=0$, while all other positions are equal to $M_{i}[1]$.

Type 2: There exists a symbol $M_{i}\left[j_{0}\right]$, different from $M_{i}[1]$, such that we can replace all the symbols $M_{i}[j]$, different from $M_{i}\left[j_{0}\right]$, with holes. All the symbols of the array are now contained in $M_{i}\left[j_{0}\right]$. Note that in this case $M_{i}^{\prime}[j]=\diamond$, for all $M_{i}^{b}[j]=1$.

One can see that, in an array, no substitution is necessary in case all of it's symbols are already equal. Due to the $d$-validity condition, the following remarks are straightforward:

Remark 2 In $d$ consecutive arrays, only one Type 2 replacement is possible.

Remark 3 If for some $k, 1 \leq k \leq q$, we have $M_{j_{0}}^{b}[k]=M_{j_{1}}^{b}[k]=0$, where $0<j_{1}-j_{0} \leq d$, then one cannot apply Type 1 replacements to both $M_{j_{0}}$ and $M_{j_{1}}$.

After these basic remarks let us solve Problem 3 .

To begin with, define the boolean variables $x_{i}$, for $i \in\{1, \ldots, m\}$, which are true, $x_{i}=1$, if and only if we apply a Type 2 replacement to $M_{i}$ in order to reach a valid solution for the problem. Then, for each $d$ consecutive arrays of $M$, say $M_{i}, \ldots, M_{i+d-1}$, we show how to construct a boolean formula, denoted $\phi_{i}$, involving some of the variables $x_{i}$ defined above. All these formulas will be the conjunction of at most 2 clauses. Finally, we derive a new formula $\phi_{M}$ from all these small formulas, and show that there exists an assignment of the variables $x_{i}$ with $i \in\{1, \ldots, m\}$, that makes $\phi_{M}$ true if and only if Problem 3 has a solution. A solution for the Problem can be derived according to the truth values of the variables $x_{i}$, where $i \in\{1, \ldots, m\}$.

First, we consider $d$ consecutive arrays, $M_{i}, \ldots, M_{i+d-1}$, all with the same number of elements $q$. Also, we assume $q \geq 2$, as the case when $q=1$ is trivial, since no replacement takes place. 
Remark 4 Let us assume that for some $k$ with $1 \leq k \leq q$, we have $M_{j_{1}}^{b}[k]=M_{j_{2}}^{b}[k]=M_{j_{3}}^{b}[k]=$ 0 , where $j_{1}<j_{2}<j_{3}$ and $j_{3}-j_{1}<d$. No matter the replacements we apply, one is not able to obtain a sequence that verifies both the periodicity and the $d$-validity condition.

Indeed, according to Remark 2 we can apply only one Type 2 and two Type 1 replacements to these three arrays. Making use of Remark 3, we get that the problem has no solution.

Similar arguments show that in order to apply a Type 2 replacement to an array $M_{j}$, for some $j \in\{i, \ldots, i+d-1\}$, the following conditions must hold:

- if there exist $k$ with $1 \leq k \leq q$, and $j_{1}, j_{2} \in\{i, \ldots, i+d-1\}$ with $j_{1}<j_{2}$, such that $M_{j_{1}}[k]=M_{j_{2}}[k]=0$, then $j \in\left\{j_{1}, j_{2}\right\}$;

- if there exist $k$ with $1 \leq k \leq q$ and a unique $j_{1} \in\{i, \ldots, i+d-1\}$ such that $M_{j_{1}}[k]=0$, then $j=j_{1}$.

Further, suppose that we have to apply a Type 2 replacement to the array $M_{j}$, for some $j \in\{i, \ldots, i+d-1\}$, and assume that exists $k$ such that $M_{j}^{b}[k]=1$. It follows that $M_{\ell}^{b}[k] \neq 0$, for all $\ell \in\{i, \ldots, i+d-1\}$. Indeed, if an $\ell$ exists, such that $M_{\ell}^{b}[k]=0$, then after the array $M_{j}$ is transformed using a Type 2 replacement, we can only apply a Type 1 replacement to the array $M_{\ell}$. Since, if we apply these two replacements it follows that $M_{j}^{\prime}[k]=M_{\ell}^{\prime}[k]=\diamond$, we get a contradiction with the $d$-validity condition.

Now, for a $k$ with $1 \leq k \leq q$, let:

$$
L_{i}(k)=\left\{\begin{array}{l}
\left\{j \mid j \in\{i, \ldots, i+d-1\}, M_{j}^{b}[k]=0\right\}, \text { if the set is nonempty } \\
\{1, \ldots, m+1\}, \text { otherwise. }
\end{array}\right.
$$

Moreover, denote by $L_{i}=\bigcap_{k \in\{2, \ldots, q\}} L_{i}(k)$. Following the remarks, if we have $3 \leq\left|L_{i}(k)\right| \leq$ $d$, for some $k$, then the problem has no solution. Also, if $\left|L_{i}\right|=m+1$ then no replacements are necessary in the arrays $M_{i}, \ldots, M_{i+d-1}$. Thus, the only cases one should analyze are $\left|L_{i}\right| \in$ $\{0,1,2\}$. We analyze each of this cases under the assumption that $3 \leq\left|L_{i}(k)\right| \leq d$ is false for all $j$.

Case 1. $\left|L_{i}\right|=0$, thus, $L_{i}=\emptyset$.

Assume $q \geq 3$. If there exist $k, 1 \leq k \leq q$, and $j_{1}, j_{2} \in\{i, \ldots, i+d-1\}$ with $j_{1} \neq j_{2}$, such that $M_{j_{1}}^{b}[k]=M_{j_{2}}^{b}[k]=0$, then we cannot apply any replacement to these arrays. Indeed, following Remarks 2 and 3 we assume without loss of generality that we apply a Type 2 replacement to $M_{j_{1}}$ and a Type 1 replacement to $M_{j_{2}}$. Since $L_{i}=\emptyset$, it follows that there exist $j_{3} \in\{i, \ldots, i+$ $d-1\}$ and $k^{\prime} \neq k$ with $1 \leq k^{\prime} \leq q$, such that $M_{j_{1}}^{b}\left[k^{\prime}\right]=1$ and $M_{j_{3}}^{b}\left[k^{\prime}\right]=0$. If we apply a Type 1 replacement to $M_{j_{3}}$, then we have holes at position $k^{\prime}$ of both $M_{j_{1}}$ and $M_{j_{3}}$. If we apply a Type 2 replacement to $M_{j_{3}}$, then we have holes at position 1 of both $M_{j_{2}}$ and $M_{j_{3}}$. A contradiction in both cases.

Now assume that the there is no $k, 1 \leq k \leq q$, and $j_{1}, j_{2} \in\{i, \ldots, i+d-1\}$ with $j_{1} \neq j_{2}$, such that $M_{j_{1}}^{b}[k]=M_{j_{2}}^{b}[k]=0$. If there exist $k_{1}$ and $k_{2}$ with $1 \leq k_{1}, k_{2} \leq q$ and $k_{1} \neq k_{2}$, and $j_{1}$ and $j_{2}$ with $j_{1}, j_{2} \in\{i, \ldots, i+d-1\}$ and $j_{1} \neq j_{2}$, such that $M_{j_{1}}^{b}\left[k_{1}\right]=M_{j_{2}}^{b}\left[k_{2}\right]=0$, then $M_{j_{1}}^{b}\left[k_{2}\right]=M_{j_{2}}^{b}\left[k_{1}\right]=1$. It follows that we cannot apply any Type 2 replacement to any of these arrays, and we must apply to both of them a Type 1 replacement. 
Finally, if there exists a unique array $M_{j}$ such that $M_{j}^{b}$ contains zeros we get a contradiction with $L_{i}=\emptyset$.

The case when $q=2$ leads immediately to the conclusion that the problem, in this case, has no solution.

Thus, we can state that if $L_{i}=\emptyset$ the problem has a solution only in the case when there are no two arrays that contain 0 at the same position (i.e., $\left|L_{i}(k)\right| \leq 1$ for all $k$ ), and in this case we must apply Type 1 replacements to all the arrays that contain 0 .

Case 2. $\left|L_{i}\right|=1$, let $L_{i}=\left\{j_{0}\right\}$.

In this case, the array $M_{j_{0}}^{b}$ has elements equal to 0 at every position where $M_{j}^{b}$ has also 0 , for $j \in\{i, \ldots, i+d-1\}$.

Assume that $q \geq 3$. We show that if there exists at least another array $M_{j_{1}}$, such that $M_{j_{1}}^{b}$ does not contain only 1's, then it is mandatory to apply a Type 2 replacement to $M_{j_{0}}$. Indeed, if $M_{j_{0}}^{b}[k]=0$, then $M_{j_{1}}^{b}[k]=0$ and there exists $k^{\prime}$ such that $M_{j_{0}}^{b}\left[k^{\prime}\right]=0$ and $M_{j_{1}}^{b}\left[k^{\prime}\right]=1$, since otherwise, $j_{1}$ would be in $L_{i}$ as well. It follows that we cannot apply Type 1 replacement to $M_{j 0}$.

If $q=2$, all the values $j$ such that $M_{j}^{b}$ contains a 0 are in $L_{i}$. Thus, there exists an unique array $M_{j_{0}}^{b}$ that contains exactly one 0 , and either a Type 1 or a Type 2 replacement can be applied to it.

To conclude, if more than one array $M_{j}^{b}$ contains 0 , then we must apply a Type 2 replacement to $M_{j_{0}}$ and Type 1 replacements to all the other arrays, if possible. If exactly one array contains 0 then either a Type 1 or a Type 2 replacement can be applied to it.

Case 3. $\left|L_{i}\right|=2$, let $L_{i}=\left\{j_{1}, j_{2}\right\}$.

In this case, it is easy to see that $M_{j_{1}}^{b}=M_{j_{2}}^{b}$. Using arguments similar to above one shows that when other arrays contain 0's the problem has no solution, or, otherwise, we must apply a Type 2 replacement to exactly one of the arrays $M_{j_{1}}$ or $M_{j_{2}}$ and Type 1 replacements to all the other arrays.

The above discussion shows that the set $L_{i}$ indicates the way Type 2 replacements should be applied to the arrays $M_{i}, \ldots, M_{i+d-1}$ in order to make them verify both the periodicity and the $d$-validity condition:

- if $L_{i}=\emptyset$, then the problem only has solution in the case when there do not exist two arrays that contain 0 at the same position (and, consequently, we must apply Type 1 replacements to all the arrays that contain 0 );

- if $L_{i}=\{1, \ldots, m+1\}$, then no replacement will be applied, and $\phi_{i}=1$;

- if $L_{i}=\left\{j_{0}\right\}$, then one of the following cases may occur:

- more than one array $M_{j}^{b}$ contains 0 . Then, we must apply a Type 2 replacement to $M_{j_{0}}$, Type 1 replacements to all the other arrays, if possible, and we have $\phi_{i}=x_{j}$;

- only one of the arrays $M_{i}^{b}, \ldots M_{i+d-1}^{b}$ contains 0 . Then we can apply only Type 1 replacements, and we have $\phi_{i}=1$;

- if $L_{i}=\left\{j_{1}, j_{2}\right\}$, where $j_{1}<j_{2}$, two cases may occur:

- if there exist other arrays than $M_{j_{1}}^{b}$ and $M_{j_{2}}^{b}$ that contain 0 , then the problem has no solution (we have 0's at the same position in three different arrays). We have $\phi_{i}=0$. 
- otherwise, we must apply a Type 2 replacement to exactly one of the arrays $M_{j_{1}}$ or $M_{j_{2}}$ and Type 1 replacements to all the other arrays. In this case, we set $\phi_{i}=x_{j_{1}}$ xor $x_{j_{2}}$.

Let us now assume that the arrays $M_{i}, \ldots, M_{i+d-1}$ do not have the same length. Let $s$ be the minimum number of elements of an array. We define the arrays $M_{j}^{\nabla}$ formed of only the first $s$ elements of $M_{j}$, for $j \in\{i, \ldots, i+d-1\}$. Thus, we either obtain a formula $\phi_{i}^{\nabla}$, just as above, for the arrays $M_{i}^{\nabla}, \ldots, M_{i+d-1}^{\nabla}$, or we see that the problem has no solution. If a formula can be computed, then we have $\phi_{i}^{\nabla}=x_{j}$; or, $\phi_{i}^{\nabla}=x_{j_{1}}$ xor $x_{j_{2}}$; or, $\phi_{i}^{\nabla}=1$.

In the first case, when $\phi_{i}^{\nabla}=x_{j}$, we have:

- if there exist $j_{1}, j_{2} \in\{i, \ldots, i+d-1\} \backslash\{j\}$ and $s^{\prime}>s$, such that $M_{j_{1}}^{b}\left[s^{\prime}\right]=M_{j_{2}}^{b}\left[s^{\prime}\right]=0$, the problem has no solution;

- if there exist $j_{1} \in\{i, \ldots, i+d-1\} \backslash\{j\}$ and $s^{\prime}>s$, such that $M_{j_{1}}^{b}\left[s^{\prime}\right]=0$ and $M_{j}^{b}\left[s^{\prime}\right]=1$, the problem has no solution;

- otherwise, we set $\phi_{i}=x_{j}$.

In the second case, when $\phi_{i}^{\prime}=x_{j_{1}}$ xor $x_{j_{2}}$, we have:

- if there exist $j_{1}^{\prime}, j_{2}^{\prime} \in\{i, \ldots, i+d-1\} \backslash\left\{j_{1}, j_{2}\right\}$ and $s^{\prime}>s$, such that $M_{j_{1}^{\prime}}^{b}\left[s^{\prime}\right]=M_{j_{2}^{\prime}}^{b}\left[s^{\prime}\right]=0$, the problem has no solution;

- if there exist $j_{1}^{\prime} \in\{i, \ldots, i+d-1\} \backslash\left\{j_{1}, j_{2}\right\}$ and $s^{\prime}>s$, such that $M_{j_{1}^{\prime}}^{b}\left[s^{\prime}\right]=0$ and $M_{j_{1}}^{b}\left[s^{\prime}\right]=$ $M_{j_{2}}^{b}\left[s^{\prime}\right]=1$, the problem has no solution;

- if there exist $j_{1}^{\prime} \in\{i, \ldots, i+d-1\} \backslash\left\{j_{1}, j_{2}\right\}$ and $s^{\prime}>s$, such that $M_{j_{1}^{\prime}}^{b}\left[s^{\prime}\right]=M_{j_{1}}^{b}\left[s^{\prime}\right]=0$ and $M_{j_{2}}^{b}\left[s^{\prime}\right]=1$, we set $\phi_{i}=x_{j_{1}}$;

- if there exist $j_{1}^{\prime} \in\{i, \ldots, i+d-1\} \backslash\left\{j_{1}, j_{2}\right\}$ and $s^{\prime}>s$, such that $M_{j_{1}^{\prime}}^{b}\left[s^{\prime}\right]=0, M_{j_{1}}^{b}\left[s^{\prime}\right]=1$ and $M_{j_{2}}^{b}\left[s^{\prime}\right]=0$, we set $\phi_{i}=x_{j_{2}}$;

- otherwise, we set $\phi_{i}=x_{j_{1}}$ xor $x_{j_{2}}$.

Finally, when $\phi_{i}^{\prime}=1$, we have:

- if there are 0's in more than one of the truncated arrays then it is mandatory to apply only Type 1 replacements to these arrays. If there exist a $s^{\prime}>s$ and $j_{1}, j_{2} \in\{i, \ldots, i+d-1\}$ such that $M_{j_{1}}^{b}\left[s^{\prime}\right]=M_{j_{2}}^{b}\left[s^{\prime}\right]$ we set $\phi_{i}^{\prime}=0$, and otherwise we leave $\phi_{i}^{\prime}=1$.

- if exactly one of the truncated arrays contains 0 's, let us denote it by $M_{j}^{b}$.

- If there exist $j_{1}, j_{2} \in\{i, \ldots, i+d-1\} \backslash\{j\}$ and $s^{\prime}>s$, such that $M_{j_{1}}^{b}\left[s^{\prime}\right]=M_{j_{2}}^{b}\left[s^{\prime}\right]=0$, then the problem has no solution.

- Assume that the above case does not hold. If there exists $j_{1} \in\{i, \ldots, i+d-1\} \backslash\{j\}$ and $s^{\prime}>s$, such that $M_{j_{1}}^{b}\left[s^{\prime}\right]=0$ and $M_{j}^{b}\left[s^{\prime}\right]=0$, then we must apply a Type 2 replacement to $M_{j}$, and set $\phi_{i}=x_{j}$.

- Otherwise, we set $\phi_{i}=1$. 


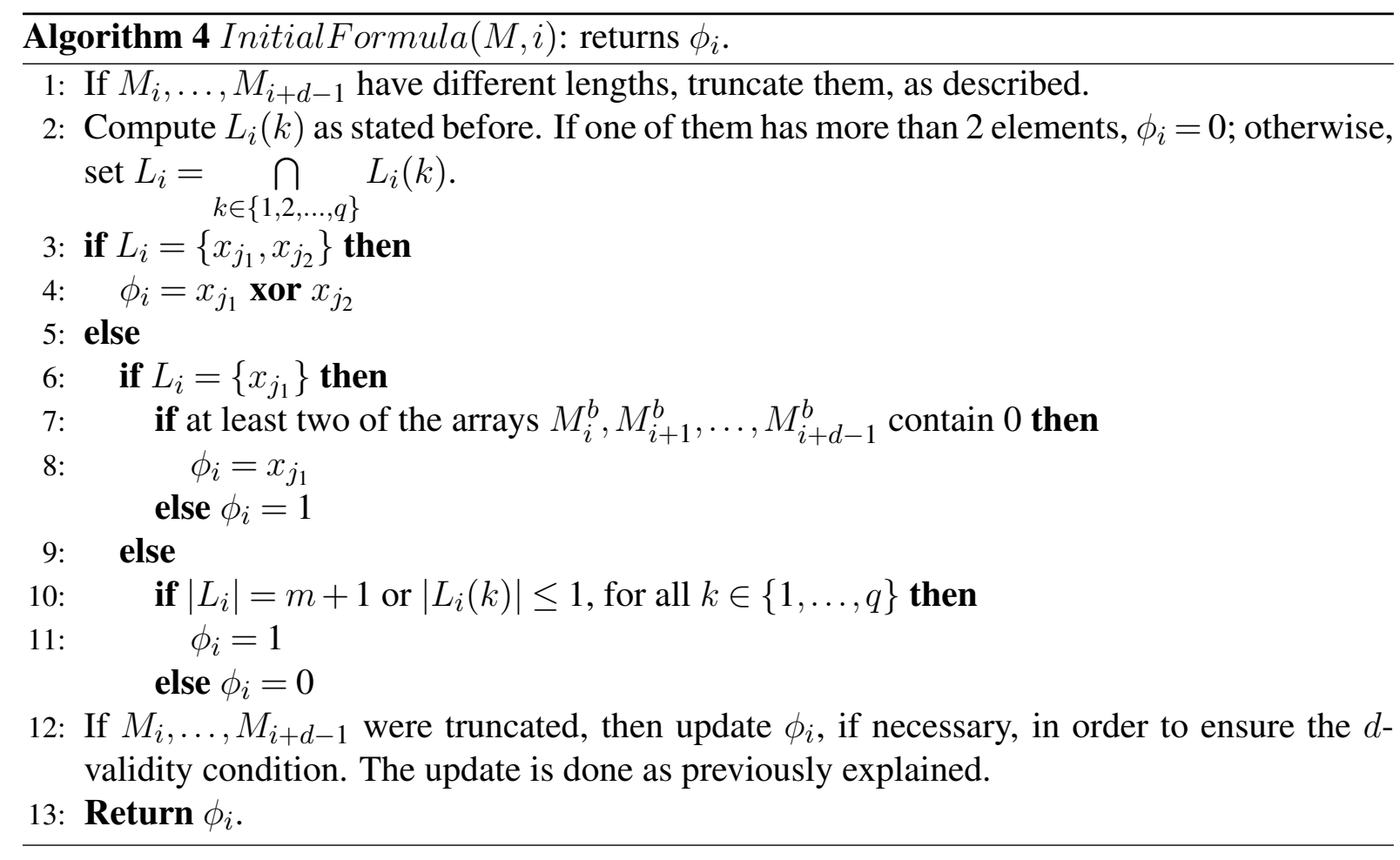

- if no 0's are in the truncated arrays, let $s^{\prime}$ be the minimum number such that there exists $j \in\{i, \ldots, i+d-1\}$ with $M_{j}^{b}\left[s^{\prime}\right]=0$. We keep only the first $s^{\prime}$ symbols of any array $M_{j}$, with $j \in\{i, \ldots, i+d-1\}$, that has at least $s^{\prime}$ symbols and try to obtain a formula for these arrays. The arrays $M_{j}^{b}$, with $j \in\{i, \ldots, i+d-1\}$, are truncated accordingly. Clearly, the only 0 's that appear in the newly truncated arrays $M_{j}^{b}$ with $j \in\left\{i, \ldots, i_{d}-1\right\}$, can only be placed at the last positions in these arrays. If there is more than one array containing 0 , then the formula we obtain for these arrays is different from 1 . Then, we repeat the analysis described above, for the original arrays.

Assume that in each formula $\phi_{i}=x_{j_{1}}$ xor $x_{j_{2}}$ we have $j_{1}<j_{2}$. In this setting, $x_{j_{1}}$ is said to be the first variable of the formula, while $x_{j_{2}}$ is the second one. If the formula consists of a single variable $\phi_{i}=x_{j_{1}}$, then this variable is said to be both the first and the second one, for uniformity.

Algorithm 4 summarizes the ideas above.

Remark 5 We conclude with some simple remarks:

- Arguments similar to the above show that if a variable $x_{i}$ does not appear in any of the $\phi_{j}$ formulas, where $1 \leq j \leq m-d+1$, then in all the solutions of the problem we can have a Type 1 replacement to array $i$. Indeed, each Type 2 replacement to such an array can be canceled, and a Type 1 replacement can be performed instead.

- Moreover, if $x_{i}$ appears in one of the formulas, then there exists an array $j$, with $0<|j-i|<d$ and a position $k$, such that $M_{i}^{b}[k]=0$ and $M_{j}^{b}[k]=0$. Thus, $M_{i}[k]$ can not be replaced with a hole in array $i$, and all symbols different from $M_{i}[k]$ should be replaced when a Type 2 replacement is performed to this array.

-Also, if $\phi_{i}=x_{j}$ or $\phi_{i}=x_{j^{\prime}}$ xor $x_{j}$ with $j^{\prime}<j$, then formulas $\phi_{i+1}, \ldots, \phi_{j+d}$ can only have one 


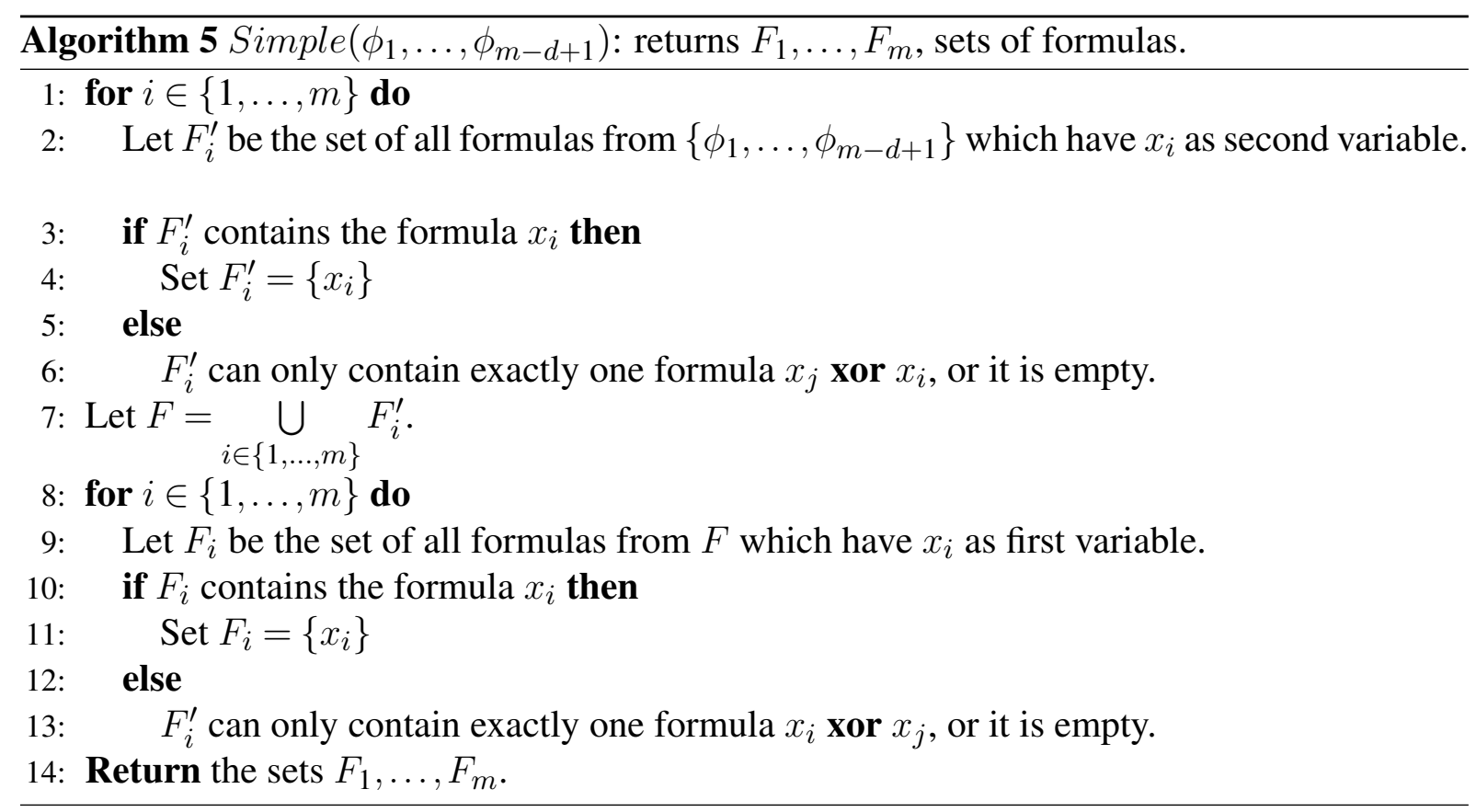

of the following forms: $x_{j^{\prime}}$ xor $x_{j}, x_{j}, 1,0, x_{j}$ xor $x_{k}$, or $x_{k}$, for some $k>j$ and $|k-i| \geq d$. Once a new variable $x_{k}$ appears in one of these formulas, the first two possibilities mentioned above cannot occur anymore.

It only remains to show how one can construct $\phi_{M}$. It is obvious that, in order to transform $M$ into a periodic $d$-valid sequence we must transform each subsequence of $M$, consisting of $d$ consecutive arrays, into a periodic $d$-valid sequence. Thus, all formulas $\phi_{i}$ should be satisfied, and if any formula is equal to 0 , then the problem has no solution. Let us assume that all the formulas are different from 0 . However, $\phi_{M}$ cannot be just the conjunction of all these formulas, since we may get into a situation in which two variables $x_{j_{1}}$ and $x_{j_{2}}$, appearing in different formulas $\phi_{i_{1}}$ and $\phi_{i_{2}}$, respectively, for which $\left|j_{1}-j_{2}\right| \leq d-1$, are both set to 1 . Furthermore, one should check if we can apply a Type 2 replacement to an array $M_{i}$ and Type 1 replacements to the $d-1$ arrays that precede it and the $d-1$ arrays that succeed it.

We proceed as follows:

First, we run Simple $\left(\phi_{1}, \ldots, \phi_{m-d+1}\right)$, described in Algorithm 5, to ensure that each variable is the first, respectively last, in at most one formula.

Second, we simplify even more the formulas by eliminating the variables that cannot be true. More precisely, we detect the arrays $i$ in which we should apply a Type 2 replacement, but we cannot do this because of the Type 1 replacements on the $d-1$ arrays that precede and the $d-1$ arrays that succeed it (and we set $x_{i}=0$ ). This is done by Filter $\left(F_{1}, \ldots, F_{m}\right.$ ), described in Algorithm 6, where $F_{1}, \ldots, F_{m}$ represent sets of formulas whose first variables are $x_{1}, \ldots, x_{m}$. Note that, each $F_{i}$ contains at most one formula. Moreover, this function computes a series of equalities $x_{i}=x_{i}^{t}$, for some $i \in\{1, \ldots, m\}$ and $s \in\{1, \ldots, q\}$, that indicate which symbol should not be transformed into a $\diamond$ when a Type 2 replacement is applied to $M_{i}$. This information is needed in order to effectively produce a solution for the problem. Finally, remark that Algorithm 6 also ensures that we cannot perform a Type 2 replacement to an array to which a Type 1 replacement was necessary, according to the analysis made in Case 1 previously 


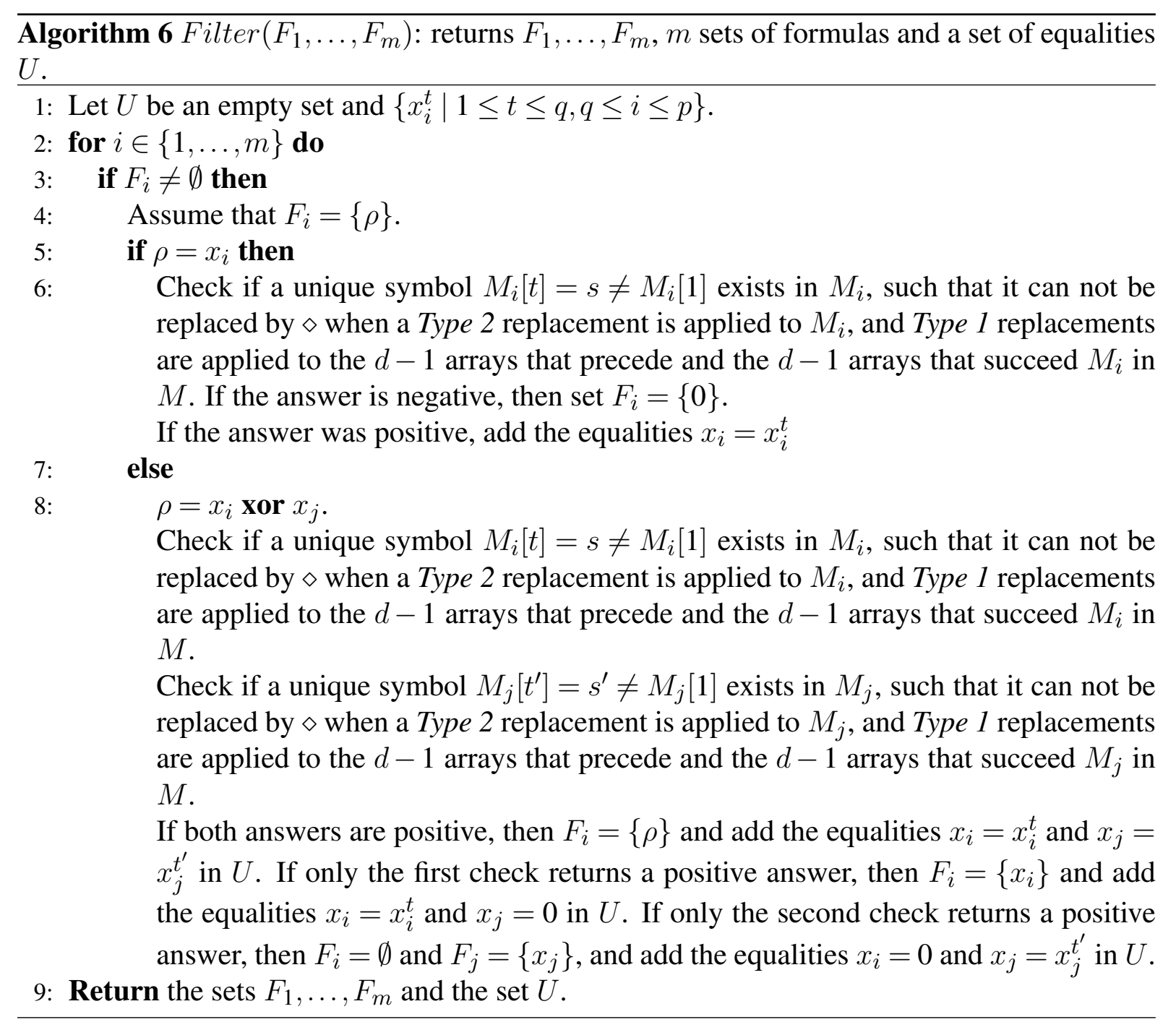

discussed.

In the end, starting from the simplified list of formulas we can construct $\phi_{M}$ by running Formula $\left(\phi_{1}, \ldots, \phi_{m-d+1}\right)$, described in Algorithm 7 .

The soundness of the three Algorithms is straightforward, since we just modify the list of formulas in order to eliminate the situations mentioned above.

It is not hard to see, according to the above remarks, that Problem 3 has a solution for the input sequence of arrays $M$ if and only if there exists a truth assignment for the variables $x_{1}, \ldots, x_{m}$ that makes $\phi_{M}$ equal to 1 . Clearly, instead of each formula $x_{i}$ xor $x_{j}$ that appears in $\phi_{M}$ we can write the formula $\left(x_{i} \vee x_{j}\right) \wedge\left(\overline{x_{i}} \vee \overline{x_{j}}\right)$. Hence, $\phi_{M}$ is a formula in 2-Conjunctive Normal Form. Thus, one can solve it efficiently by an algorithm that solves the 2-CNF-SAT Problem in linear time [1].

However, a more careful analysis shows that, the special form of the formula $\phi_{M}$, as it results from Algorithm 7, allows us to decide whether it is satisfiable or not far more easily: the only case when $\phi_{M}$ is not decidable is when it equals 0 . Otherwise, we can find a truth assignment that makes $\phi_{M}=1$ in the following way: if the clause $\left(x_{i}\right)$ is present in $\phi_{M}$ then we assign $x_{i}=1$, delete these variables from $\phi_{M}$, and for the rest of the variables that appear 


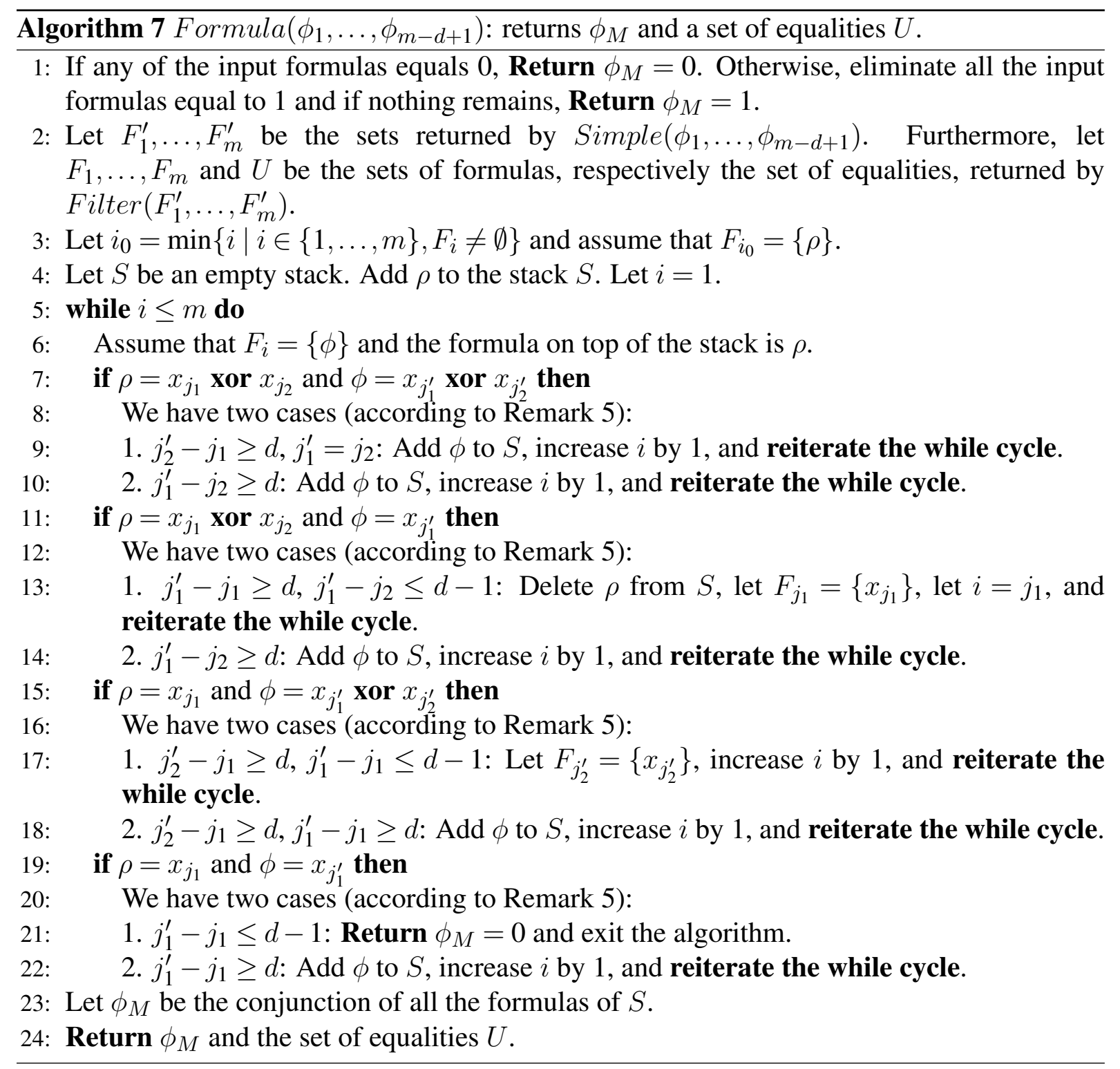

in the formula we choose an assignment that makes all the clauses true. The latter part can be done quite easily:

- if $\left(x_{i}\right.$ xor $\left.x_{j}\right)$ is the first clause that appears in $\phi_{M}$, then we set $x_{i}=b$, for $b \in\{0,1\}$ and $x_{j}=\bar{b}$;

- as we have seen, $x_{j}$ can only appear in the second clause;

- if the second clause is $x_{j} \operatorname{xor} x_{t}$, then we set $x_{t}=\overline{x_{j}}$, and try to satisfy the rest of the clauses in a similar manner;

- if the second clause is $x_{j^{\prime}}$ xor $x_{t}$ with $j^{\prime}, t>j$, then we set $x_{j^{\prime}}=b$ and $x_{t}=\bar{b}$, where $b \in\{0,1\}$, and try to satisfy the rest of the clauses in a similar manner.

Thus, a solution for Problem 3 is given by Algorithm 8 . 


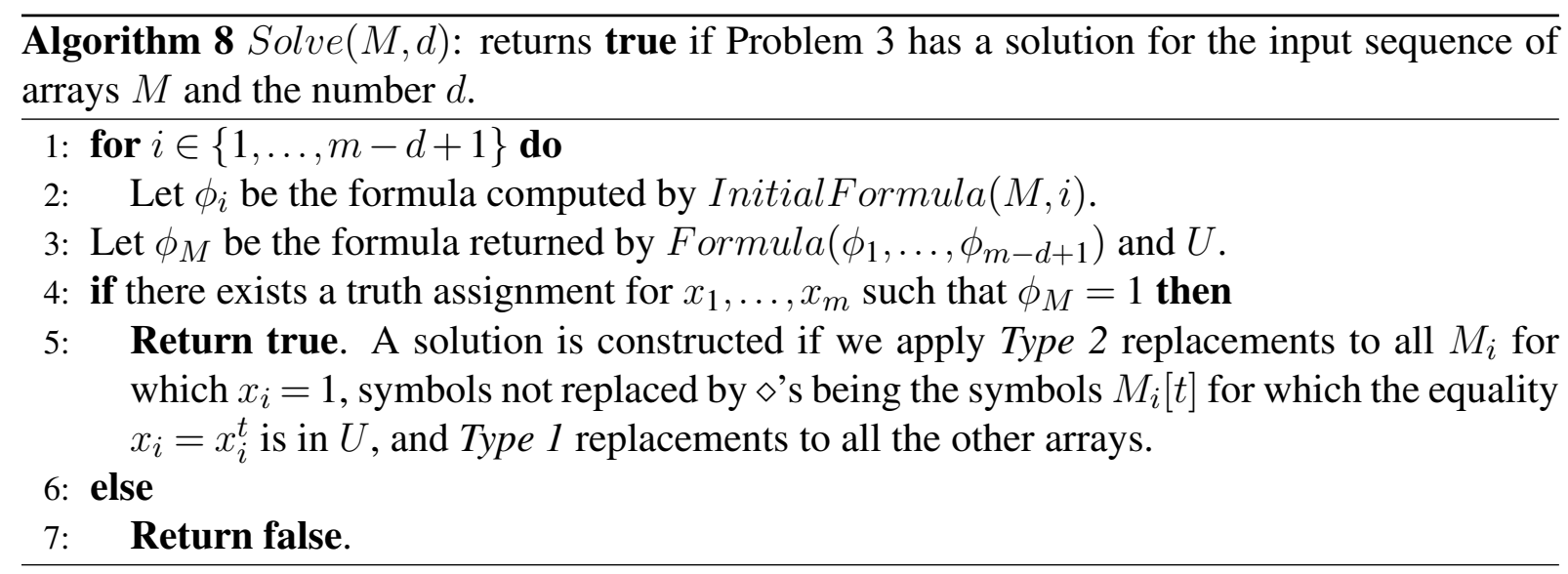

\section{Complexity aspects}

The fact that Algorithm 8 solves Problem 3 follows from the previous discussions. However, in order to show the complexity part we discuss several implementation details of the aforementioned algorithms.

To begin with, we make the usual assumption (for the RAM-model) that the time needed to read (access the memory location containing) a symbol from $V$ or to compare two such symbols is constant. The size of an input of Problem 3 is proportional with the total number of elements of the arrays that form the sequence $M$, times the memory needed to represent a symbol of $V$ (denoted here size $(V)$ ), plus the number of bits needed to represent $d$. In other words, the size of the input is $\mathcal{O}(\operatorname{size}(V) m q+\log d)$.

We show that the formula $\phi_{M}$ is constructed in linear time and, since the formula has $\mathcal{O}(m q)$ clauses and variables, deciding its satisfiability, and finding an assignment, for the variables, that makes $\phi_{M}=1$, also takes linear time. Hence, deciding whether Problem 3 has a solution, and effectively finding one, is done in $\mathcal{O}(\mathrm{mq})$ time. The proof consists in several observations that ensure that each of the previously described procedures is implemented in $\mathcal{O}(m q)$ time.

Note that the sets used in the previous section's algorithms are always implemented as queues. Moreover, the sequence $M^{b}$ is constructed in time $\mathcal{O}(m q)$, canonically.

Let us now investigate the time complexity for Algorithm 4 . In particular, we show how one computes efficiently the sets $L_{i}$ and the formulas $\phi_{i}$, for all $i$ :

- to this end, we fix $i \in\{1, \ldots, m-d+1\}$ and we define, for each $k \in\{1, \ldots, q\}$, an ordered queue $Q_{k}$ that contains, at the moment when $L_{i}$ is computed, the values $\left\{i_{1}, \ldots, i_{t}\right\}$, such that $M_{i_{j}}^{b}[k]=0$ and $0 \leq i_{j}-i<d$, for all $j \in\{1, \ldots, t\}$. Furthermore, we define, for each $k \in$ $\{1, \ldots, q\}$, a variable $p_{k}$ that stores the cardinality of the queue $Q_{k}$.

- if one of the variables $p_{k}$ is greater than or equal to 3 , when $L_{i}$ is computed, we decide that $\phi_{i}=0$. Otherwise, we determine in $\mathcal{O}(q)$ time the elements that appear in all the non-empty queues $Q_{k}$, since these queues have at most 2 elements, and return these elements as the set $L_{i}$. Moreover, we compute in $\mathcal{O}(q)$ time the formula $\phi_{i}$, as described in Algorithm 4 ,

- note that, when $i=1$, we construct the queues $Q_{k}$ with $k \in\{1, \ldots, q\}$, by simply traversing the arrays $M_{1}, \ldots, M_{d}$, which takes $\mathcal{O}(d q)$ time. Then, in order to update the queues, when we move from computing $L_{i}$ and the formula $\phi_{i}$, to computing $L_{i+1}$ and the formula $\phi_{i+1}$, we just have to delete the minimum element from all these queues, if this element equals $i$, and add to 
the queue $Q_{k}$ the element $i+d$, for all values of $k$ such that $M_{i+d}^{b}[k]=0$. Again, this operations take $\mathcal{O}(q)$ time;

- following the above, the time needed to compute all formulas $\phi_{i}$, for $i \in\{1, \ldots, m-d+1\}$, is $\mathcal{O}(m q)$.

It is not hard to see that Algorithm 5 runs in $\mathcal{O}(m)$ time.

Let us now analyze the time complexity of Algorithm 6 .

- the verifications from the iterative block of Algorithm 6 can be performed efficiently using a strategy similar to the one described for Algorithm 4.

- we start with the remark that a symbol can be replaced by a hole in array $M_{i}$ using a Type 2 replacement, if and only if none of the $d-1$ arrays that precede it, nor the $d-1$ arrays that succeed it, have 0 at that position. Thus, we define, for each $k \in\{1, \ldots, q\}$, two ordered queues, $Q_{k}^{\prime}$ and $Q_{k}^{\prime \prime}$, that contain, at the moment when $F_{i}$ is checked, the values $\left\{i_{1}, \ldots, i_{t}\right\}$, such that $M_{i_{j}}^{b}[k]=0$ and $0<i_{j}-i<d$, and, respectively, the values $\left\{i_{1}^{\prime}, \ldots, i_{s}^{\prime}\right\}$, such that $M_{i_{\ell}^{\prime}}^{b}[k]=0$ and $0<i-i_{\ell}^{\prime}<d$, for all $j \in\{1, \ldots, t\}$ and $\ell \in\{1, \ldots, s\}$. These queues can be computed and updated just as in the previous case and none of them contains more than 2 elements at a given moment. For our verifications, it suffices to check if the set $\left\{M_{i}[j] \mid\right.$ with $Q_{j}^{\prime}$ or $Q_{j}^{\prime \prime}$ not empty $\}$ contains exactly one symbol. If so, the verification returns a positive answer. Otherwise, it returns a negative one. Clearly, the time needed to do this is $\mathcal{O}(m q)$;

- according to this, the total running time needed by Algorithm 6 is $\mathcal{O}(m q)$.

It is not hard to see that Algorithm 7 runs in $\mathcal{O}(m)$ time. We notice that if any point $\phi$ is the formula on top of the stack $S$, and $x_{j}$ is the second variable of $\phi$, then all the variables $x_{i}$ that appear in the formulas from $S$ verify $i<j$.

Summarizing the above, it follows that Algorithm 8 works in $\mathcal{O}(m q)$ time. Therefore, the main result of this section is the following:

Lemma 3.2 Problem 3 can be decided in linear time. A solution for this Problem can also be obtained in the same time complexity, using Algorithm 8.

\subsection{The main problem}

In the following, Problem 2 is solved using the previously discussed solution of Problem 3 , in time $\mathcal{O}(|w|)$, where the constant hidden by the $\mathcal{O}$-denotation does not depend on either $d$ nor $p$.

Let us first analyze the case when $d \leq p$. Define the sequence of $p+d-1$ arrays $M$. For $i \leq p$, the array $M_{i}$ contains all symbols $w[x]$ with $x \geq 1$ and $x \equiv i \bmod p$. For $j$, where $1 \leq j \leq d-1$, the array $M_{p+j}$ contains the symbols $w[p+x]$ with $x \geq 1$ and $x \equiv j \bmod p$. Finally, construct the formula $\phi_{M}$ as explained in the previous section. Note that in the case when $d=1$ the formula is canonically equal to 1 .

We associate to each $M_{i}$ a variable $x_{i}$, as previously explained, for all $i \in\{1, \ldots, p+d-1\}$. Note that if $j \equiv i(\bmod p)$ and $j>i$ then the variables $x_{i}$ and $x_{j}$ are not independent, as substituting some of the symbols of the array $M_{i}$ with holes implies a substitution of the symbols of $M_{j}$ with holes. This is because $M_{j}$ contains only symbols of $w$ that appear also in $M_{i}$. To capture the dependency in our formula, we use the set of equalities $U$, produced by algorithm Filter, and we extend it to obtain a new set of equalities $U^{\prime}$, as follows. Generally, for some $i \leq p$ the formulas $\bar{x}_{i}=x_{i}^{1}, \bar{x}_{i+p}=x_{i+p}^{1}$ (i.e., applying Type 2 replacements on the arrays $i$ or $i+p$ means that the first symbol is not preserved), and $\bar{x}_{i}^{1} \neq x_{i+p}^{1}$ when $M_{i}[1] \neq M_{i+p}[1]$, will 
be added to $U^{\prime}$. Assume now that we have $i \leq p$ and $x_{i}=x_{i}^{\ell}$ and $x_{i+p}=x_{i+p}^{\ell^{\prime}}$ in $U$; we also add to $U^{\prime}$ the equalities $\bar{x}_{i}^{\ell}=x_{i+p}^{\ell^{\prime}}$ if $M_{i}[\ell] \neq M_{i+p}\left[\ell^{\prime}\right], \bar{x}_{i}^{\ell}=x_{i+p}^{1}$ if $M_{i}[\ell] \neq M_{i+p}[1]$, and $\bar{x}_{i}^{1}=x_{i+p}^{\ell^{\prime}}$ if $M_{i}[1] \neq M_{i+p}\left[\ell^{\prime}\right]$ (these formulas say that the replacements on array $i$ and on array $i+p$ must leave the same symbol unchanged). Next, we conjunct $\phi_{M}$ and all formulas in $U^{\prime}$, to obtain a new formula $\phi_{M}^{\prime}$.

Next, we make the conjunction between $\phi_{M}$ and all the formulas in $U^{\prime}$, and obtain a new formula $\phi_{M}^{\prime}$ in 2-Conjunctive Normal Form.

Problem 2 has a solution if and only if there exists a truth assignment for the variables $\left\{x_{1}, \ldots, x_{p+d-1}\right\} \cup\left\{x_{i}^{t} \mid 1 \leq t \leq q\right.$ and $\left.1 \leq i \leq p\right\}$ that gives $\phi_{M}^{\prime}=1$. A solution is constructed applying Type 2 replacements to all $M_{i}$ with $x_{i}=1$ and Type 1 replacements to all other arrays, followed by corresponding replacements in the input word. For the Type 2 replacements, the symbols not replaced by $\diamond$ 's are the symbols $M_{i}[t]$, where the equality $x_{i}=x_{i}^{t}$ is in $U$.

Clearly, if $d \leq p+1$, then it is quite easy to implement all of the previous steps in linear time, with respect to the length of the input word $w$. The time needed to solve the problem does not depend on neither $d$ nor $p$.

The case when $d>p$ is very simple. First we define the sequence of arrays $M$, such that, for $i \leq p, M_{i}$ contains all the symbols $w[x]$ with $x \geq 1$ and $x \equiv i \bmod p$.

Some of the symbols of the arrays must be replaced in order to ensure that, for every $M_{i}$, there exists a symbol $s_{i}$ that contains all the symbols of the array.

Let $M_{i_{0}}$ be the first array that contains at least two different symbols. Although several symbols of this array must be replaced with holes, note that it is not the case for any two consecutive symbols, since this violates the $d$-validity condition. It follows that there are at most two possibilities to choose the symbol that will not be replaced by $\diamond$ in the array: if it contains two different symbols, one of them must be replaced with hole, and the other not. Assume now that we choose to keep a symbol $a$ in this array and we replace all other symbols with $\diamond$ 's, such that the aforementioned condition is fulfilled. This replacement determines on each array $M_{i}$ of $M$ a symbol $a_{i}$ that will be kept unchanged. Thus, we already determined all the symbols of $M$ that should be replaced with $\diamond$.

We only have to check whether the word that we obtain is $d$-valid. If yes, we have a solution for the problem, and otherwise, we have to try another choice for the symbol $a$ in the $M_{i}$ 's. Finally, if by using this strategy, we cannot find a valid replacement, the problem has no solution.

It is not hard to see that the time needed to solve the problem, in this case, is also $\mathcal{O}(|w|)$, and does not depend on neither $d$ nor $p$. Clearly, this time is optimal. In conclusion, we have shown Theorem 3.1 .

This theorem improves the result in [5], where Problem 2 was solved in time $\mathcal{O}(n d)$. It is worth noting that our approach is completely different from the one in the cited paper.

\subsection{Looking for the optimal number of holes}

In this final section we approach an optimization problem related to Problem 2 ;

Problem 4 Given a word $w \in V^{*}$ and two positive integers $d$ and $p$, both less than $|w|$, construct a $p$-periodic $d$-valid partial word contained in $w$, and having a minimum number of holes. 
The solution of this problem is based on the solution of Problem 2 we presented in the previous section. Let us assume, without losing generality, that Problem 2 has a solution for the input word $w$ and the numbers $d$ and $p$. As a first step we construct for the word $w$ and the integer $p$, the sequence of arrays $M$, using Algorithm 7, the formula $\phi_{M}$, and the set of equalities $U$.

In addition to Remark 5 note that if a variable $x_{i}$ does not appear in $\phi_{M}$ and a Type 2 replacement can be applied to the array $i$ in a correct solution of Problem 2 (that is the algorithm Filter did not set $x_{i}=0$ ), then both the $d-1$ arrays that precede the array $M_{i}^{b}$ and the $d-1$ arrays that succeed it contain only 1's. For a solution of Problem 2 it is not really important if we apply a Type 1 or a Type 2 replacement to this array, but in the case of the associated optimization Problem 4, when we want to have only a minimal number of holes, it is important to use, on array $i$, a replacement that leads to such a solution. Therefore, if the symbol that occurs the most times in array $i$ is $M_{i}[t]$, and this is not the first symbol of the array, then we update $\phi_{M}$ by making the conjunction with $x_{i}$ and add to $U$ the equality $x_{i}=x_{i}^{t}$. Clearly, in $\phi_{M}$ there exists no variable $x_{j}$ such that $|j-i|<d$.

For simplicity we assume that all variables $x_{i}$ with $i \in\{1, \ldots, p+d-1\}$, that do not appear in $\phi_{M}$, are set to 0 .

We can associate with each variable $x_{i}$ two values, denoted $v[i][1]$ and $v[i][2]$, for $i \in$ $\{1, \ldots, p\}$, such that $v[i][1]$ equals the number of holes that are inserted in the array $M_{i}$ if we set $x_{i}=1$, and $v[i][2]$ equals the number of holes that are inserted in the array $M_{i}$ if we set $x_{i}=0$. All these values can be computed in linear time, using the set $U$. Clearly, the solution of Problem 4 consists in finding a truth assignment of the variables $x_{1}, \ldots, x_{p}, x_{p+1}, \ldots, x_{p+d-1}$ such that the formula $\phi_{M}$ becomes true, and the value of the sum $\sum_{i \in\{1, \ldots, p\}}\left(x_{i} v[i][1]+(1-\right.$ $\left.\left.x_{i}\right) v[i][2]\right)$ is minimum.

The truth value of the variable $x_{p+j}$, for $1 \leq j \leq d-1$, associated with the array $M_{p+j}$, is not independent from the truth value of the variable $x_{j}$, as we already explained. For the first $d$ arrays in the sequence $M, \phi_{M}$ contains a unique a formula $\phi_{1}=1, \phi_{1}=x_{i}$ or $\phi_{1}=x_{i_{1}}$ xor $x_{i_{2}}$, such that $i, i_{1}, i_{2} \in\{1, \ldots, d\}$. This formula is obtained by Algorithm 4 or was inserted in the step we mentioned above (we identified an array $i$ to which it does not matter which type of replacement we apply, and applying a Type 2 replacement leads to a lower number of holes). In each case we obtain two new formulas from $\phi_{M}$ :

- If $\phi_{1}=1$, then we apply to all arrays $M_{1}, \ldots, M_{d}$ only Type 1 replacements. Thus, we can determine easily the truth values of the variables $x_{p+1}, \ldots, x_{p+d-1}$. We rewrite the formula $\phi_{M}$ according to the values that we have determined, and we have now a new formula $\phi_{M}^{\prime}$ in which only variables $x_{j}$ with $j \leq p$ appear. We also set $\phi_{M}^{\prime \prime}=0$.

- If $\phi_{1}=x_{i}$, then we perform Type 1 replacements to all first $d$ arrays except for $M_{i}$, where we apply a Type 2 replacement. Again, we easily determine, using the set of equalities $U$, the truth values for the variables $x_{p+1}, \ldots, x_{p+d-1}$. Finally, we rewrite the formula $\phi_{M}$ according to the values that we have determined, and we have now a new formula $\phi_{M}^{\prime}$ in which only variables $x_{j}$ with $j \leq p$ appear. As in the previous case, we set $\phi_{M}^{\prime \prime}=0$.

- If $\phi_{1}=x_{i_{1}}$ xor $x_{i_{2}}$, then we have, of course, two new cases, according to the variable which is set to 1 . But, in both cases we can determine, using the set of equalities $U$, the truth values of the variables $x_{p+1}, \ldots, x_{p+d-1}$. However, setting one of the variables $x_{i_{1}}$ or 
$x_{i_{2}}$ to 1 may determine the truth values of some other variables $x_{j}$, with $j \leq p$, that appear in $\phi_{M}$. Consequently, we can rewrite the formula $\phi_{M}$ in two ways, according to which of the variables $x_{i_{1}}$ or $x_{i_{2}}$ was set to 1 and to the variables whose value was determined by this choice. We obtain two new formulas $\phi_{M}^{\prime}$ and $\phi_{M}^{\prime \prime}$, respectively, in which only variables $x_{j}$ with $j \leq p$ appear.

Clearly, to solve Problem 4 we can proceed as follows. First, we search for a truth assignment of the remaining variables that make $\phi_{M}^{\prime}$ true, and the sum $\sum_{i \in\{1, \ldots, p\}}\left(x_{i} v[i][1]+(1-\right.$ $\left.\left.x_{i}\right) v[i][2]\right)$ minimum compared to the other assignments that make this formula true. Next, we search for an assignment that makes $\phi_{M}^{\prime \prime}$ true and the sum minimum, compared to the other assignments that make $\phi_{M}^{\prime \prime}$ true. The answer to our problem is provided by one of these assignments, namely the one that yields the smallest sum.

Let $\rho$ denote one of the formulas $\phi_{M}^{\prime}$ and $\phi_{M}^{\prime \prime}$. We already have an assignment of the variables $\left\{x_{1}, \ldots, x_{j}\right\}$, for a $j \geq d$, and the formula $\rho$ contains only variables from $\left\{x_{j+1}, \ldots, x_{p}\right\}$. Moreover, in an assignment that satisfies $\phi_{M}$ we must set $x_{i}=1$ for all the clauses $\left(x_{i}\right)$ that appear in $\rho$. Let us assume that $\rho$ is the conjunction of $t$ clauses, each of these clauses being of the form $x_{i_{1}}$ xor $x_{i_{2}}$, where $i_{1}<i_{2}$. Let $V$ denote a subset of $\{1, \ldots, p\}$ for which the value of $x_{i}$ was already determined and set $S=\sum_{i \in V}\left(x_{i} v[i][1]+\left(1-x_{i}\right) v[i][2]\right)$. Assume, also, that the clauses of $\rho$ are ordered according to the index of their first variable, and let $c(i)$ denote the $i^{t h}$ clause of $\rho$ with respect to this order. Let $f(i)$ and $s(i)$ denote the arrays to which the first and, respectively, the second variable of $c(i)$ are associated. For example, if $c(i)=x_{i_{1}}$ xor $x_{i_{2}}$ with $i_{1}<i_{2}$, then $f(i)=i_{1}$ and $s(i)=i_{2}$; if $c(i)=x_{i_{1}}$, then $f(i)=s(i)=i_{1}$. Of course, $f(i)>f(i-1)$, for all $i \in\{2, \ldots, t\}$.

According to the previously made remarks, the truth value of the variables that appear in $c(i)$ depend only on the values of the variables that appear in $c(i-1)$. This suggests that we can find for the variables an assignment that minimizes the sum $\sum_{j \in\{1, \ldots, p\} \backslash V}\left(x_{j} v[j][1]+\left(1-x_{j}\right) v[j][2]\right)$ in linear time, by using a dynamic programming strategy.

Let $T_{1}$ and $T_{2}$ be two arrays with $t$ elements. These arrays are defined as follows. For $i \in\{1, \ldots, t\}$ :

- If $c(i)=x_{i_{1}}$ xor $x_{i_{2}}$ then:

$-T_{1}[i]$ is the value of the sum $S+\sum_{j \in\left\{1, \ldots, i_{2}\right\} \backslash V}\left(x_{j} v[j][1]+\left(1-x_{j}\right) v[j][2]\right)$, where the truth values for the variables $x_{1}, \ldots, x_{i_{1}-1}$ were already determined, such that

$$
S+\sum_{j \in\left\{1, \ldots, i_{1}-1\right\} \backslash V}\left(x_{j} v[j][1]+\left(1-x_{j}\right) v[j][2]\right)
$$

is minimum and their values permit the assignment $x_{i_{1}}=1$ and $x_{i_{2}}=0$. If such an assignment is impossible, then $T_{1}[i]=\infty$.

$-T_{2}[i]$ is the value of the sum $S+\sum_{j \in\left\{1, \ldots, i_{2}\right\} \backslash V}\left(x_{j} v[i][1]+\left(1-x_{j}\right) v[i][2]\right)$, where the truth values for the variables $x_{1}, \ldots, x_{i_{1}-1}$ were already determined such that

$$
S+\sum_{j \in\left\{1, \ldots, i_{1}-1\right\} \backslash V}\left(x_{j} v[j][1]+\left(1-x_{j}\right) v[j][2]\right)
$$

is minimum and their values permit the assignment $x_{i_{1}}=0$ and $x_{i_{2}}=1$. If such an assignment is impossible, then $T_{2}[i]=\infty$. 
- If $c(i)=x_{i_{1}}$ then:

$-T_{1}[i]=T_{2}[i]$ and it is the value of the sum $S+\sum_{j \in\left\{1, \ldots, i_{2}\right\} \backslash V}\left(x_{j} v[j][1]+\left(1-x_{j}\right) v[j][2]\right)$, where the truth values for the variables $x_{1}, \ldots, x_{i_{1}-1}$ were already determined such that

$$
S+\sum_{j \in\left\{1, \ldots, i_{1}-1\right\} \backslash V}\left(x_{j} v[j][1]+\left(1-x_{j}\right) v[j][2]\right)
$$

is minimum.

It is rather simple to notice that these arrays are computed in linear time, hence we skip the details. Clearly, the assignment that we are looking for is the one that makes the sum $\sum_{j \in\{1, \ldots, p\} \backslash V}\left(x_{j} v[j][1]+\left(1-x_{j}\right) v[j][2]\right)$ equal to $\min \left\{T_{1}[t], T_{2}[t]\right\}$. The values assigned to the variables are also computed using dynamic programming, during the computation of the arrays $T_{1}$ and $T_{2}$.

We are now ready to state the main result of this section:

Theorem 3.3 Problem 4 can be decided in linear time. A solution for this Problem can also be obtained in the same time complexity.

Once again this result is optimal, with respect to the time complexity.

It is interesting to note that in the case when $d=1$ we can reformulate Problem 4 in a very simple way:

Problem 5 Given a word $w \in V^{*}$ and positive integer $p<|w|$, construct a $p$-periodic partial word contained in $w$, and having a minimum number of holes.

The solution that we already presented can be particularized to this case, and we get a very simple greedy solution for this problem. First, we construct for the word $w$ and the integer $p$ the sequence of arrays $M$, using Algorithm 7 , and the formula $\phi_{M}$. We obtain $\phi_{M}=1$. Then, we add to this formula all the variables $x_{i}$ with $i \in\{1, \ldots, p\}$, such that applying a Type 2 replacement to the array $i$ introduces a lower number of holes than applying a Type 1 replacement. Finally, we set all these variables to 1, and this solves the problem. In simpler words, in each of the arrays we replace with holes a minimum number of symbols such that the periodicity condition is fullfiled. The solution that we proposed for Problem 5 is also optimal with respect to the time complexity.

A final remark is that the same algorithms can be used to solve in linear time the following problem: given a word $w \in V^{*}$ and two positive integers $d$ and $p$, both less than $|w|$, construct a $p$-periodic $d$-valid partial word contained in $w$, and having a maximum number of holes.

\section{References}

[1] B. Aspvall, M. F. Plass, R. E. Tarjan, A linear-time algorithm for testing the truth of certain quantified boolean formulas. Information Processing Letters 8 (1979) 3, 121-123.

[2] F. Blanchet-Sadri, Algorithmic Combinatorics on Partial Words. Chapman \& Hall/CRC Press, 2008. 
[3] F. Blanchet-Sadri, A. R. Anavekar, Testing primitivity on partial words. Discrete Applied Mathematics 155 (2007) 3, 279-287.

[4] F. Blanchet-SADRI, A. Chriscoe, Local periods and binary partial words: an algorithm. Theoretical Computer Science 314 (2004) 1-2, 189-216.

[5] F. Blanchet-Sadri, R. Mercaş, A. Rashin, E. Willett, An Answer to a Conjecture on Overlaps in Partial Words Using Periodicity Algorithms. In: A. H. DEDIU, A. M. IOnEscu, C. MARTín-VIDE (eds.), LATA 2009. Lecture Notes in Computer Science 5457, Springer, Berlin, Germany, 2009, 188-199.

[6] C. Choffrut, J. Karhumäki, Combinatorics of Words. In: G. RozenberG, A. SALOMAA (eds.), Handbook of Formal Languages. 1, Springer, 1997, 329-438.

[7] P. Clifford, R. Clifford, Simple deterministic wildcard matching. Information Processing Letters 101 (2007) 2, 53-54.

[8] R. COLE, R. HARIHARAN, Verifying candidate matches in sparse and wildcard matching. In: STOC. 2002, 592-601.

[9] M. Crochemore, String-Matching on Ordered Alphabets. Theoretical Computer Science 92 (1992) 1, 33-47.

[10] A. Diaconu, F. Manea, C. Tiseanu, Combinatorial queries and updates on partial words. In: FCT 2009. Springer, Berlin, Heidelberg, 2009, 96-108.

[11] M. J. Fischer, M. S. PATERSON, String matching and other products. In: Complexity of Computation, SIAM-AMS Proceedings. 7, 1974, 113-125.

[12] Z. GALIL, J. I. SEIFERAS, Time-space optimal string matching. Journal of Computer and System Sciences 26 (1983), 280-294.

[13] D. E. KNUTH, The Art of Computer Programming. 1: Fundamental Algorithms, AddisonWesley, 1968.

[14] P. Leupold, Languages of Partial Words - How to Obtain Them and What Properties They Have. Grammars 7 (2004), 179-192.

[15] P. Leupold, Partial Words for DNA Coding. In: C. Ferretti, G. MAuri, C. Zandron (eds.), DNA 2004. Lecture Notes in Computer Science 3384, Springer, Berlin, Germany, 2009, 224-234.

[16] M. Lothaire, Combinatorics on Words. Cambridge University Press, 1997.

[17] F. ManeA, R. Mercaş, Freeness of partial words. Theoretical Computer Science 389 (2007) 1-2, 265-277. 